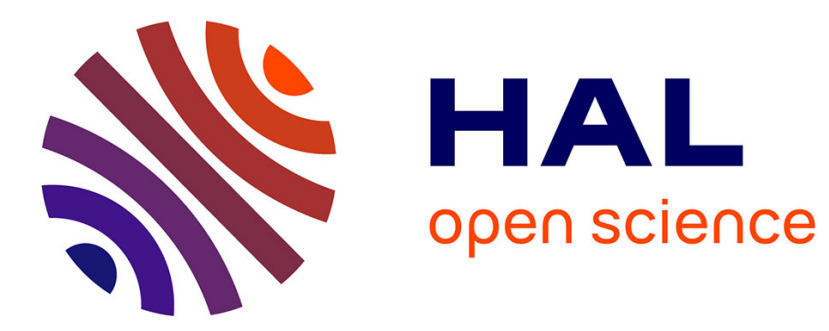

\title{
Poole-Frenkel (PF) effect high field saturation
}

\author{
R. Ongaro, A. Pillonnet
}

\section{To cite this version:}

R. Ongaro, A. Pillonnet. Poole-Frenkel (PF) effect high field saturation. Revue de Physique Appliquée, 1989, 24 (12), pp.1085-1095. 10.1051/rphysap:0198900240120108500 . jpa-00246146

\section{HAL Id: jpa-00246146 https://hal.science/jpa-00246146}

Submitted on 1 Jan 1989

HAL is a multi-disciplinary open access archive for the deposit and dissemination of scientific research documents, whether they are published or not. The documents may come from teaching and research institutions in France or abroad, or from public or private research centers.
L'archive ouverte pluridisciplinaire HAL, est destinée au dépôt et à la diffusion de documents scientifiques de niveau recherche, publiés ou non, émanant des établissements d'enseignement et de recherche français ou étrangers, des laboratoires publics ou privés. 


\title{
Poole-Frenkel (PF) effect high field saturation
}

\author{
R. Ongaro and A. Pillonnet \\ Laboratoire d'Electricité, Université Claude Bernard, 43 Boulevard du 11 Novembre 1918, 69622 Villeurbanne \\ Cedex, France
}

(Reçu le 10 novembre 1988, révisé le 27 juin 1989, accepté le 8 septembre 1989)

\begin{abstract}
Résumé. - Un effet Poole Frenkel (PF) amélioré est proposé, en utilisant une approche méthodologique rigoureuse. Le modèle choisi est détaillé précisément, l'accent étant mis explicitement sur les hypothèses sousjacentes. La référence systématique à la fonction de Fermi-Dirac permet d'établir quantitativement le concept de saturation PF. La théorie générale qui en résulte intègre, comme applications particulières, les précédentes théories PF à une dimension.
\end{abstract}

\begin{abstract}
An improved Poole Frenkel (PF) effect, based upon a rigorous methodological approach, is proposed. The chosen model is stated precisely, emphasis being made explicitly on the subtending hypotheses. A systematic reference to Fermi-Dirac function, allows to establish quantitatively the concept of PF saturation. The resulting general theory integrates, as particular applications, the previous one-dimensional PF theories.
\end{abstract}

\section{Introduction.}

The Poole Frenkel effect (labelled hereafter as PF effect), a field-enhanced free carrier density associated with a field-reduction of a coulombic potential barrier, was first proposed by Frenkel [1]. Many theoretical developments have been published on the subject from that time. Meanwhile it has been largely used as a possible interpretation of many experimental field-driven permanent non-ohmic currents.

An exhaustive analysis of the available PF theories is beyond the scope of this paper. But we believe necessary to give an outline of the basic subtending hypotheses in order to emphasize, from a user's view-point, the diversity of PF models. These assumptions are as follows.

(i) A single carrier (electron) is generally considered, referring to situations where n-type conduction is dominant.

(ii) Ohmic contacts are supposed to exist, so as to ensure that electrons swept away by the field $F$ are replenished at the proper electrode.

(iii) Any space charge, either injected or depleted, is generally ignored, whatever the field which is mainly taken as uniform. Few exceptions can be found, for example, in the works of Frank and Simmons [2], Simmons [3], or Murgatroyd [4].

(iv) Mobility is more often supposed to be fieldindependent, so that the basic, linear, drawing of current is usually made in a $\lg (I / F)$ versus $F^{1 / 2}$ plot. Such representation would be readily justified in crystalline semiconductors, or following Hill [5], in semi-crystalline materials. However, it is largely admitted, in conformity with Jonscher's [6] statement, that PF effect can be detected only in low mobility materials. For, high mobility in crystalline solids would result, in high fields, in a depletion of carriers, the re-trapping process becoming somewhat inefficient. Consequently, PF effect is often introduced as a plausible explanation of $I(V)$ curves in semi-crystalline or amorphous materials, and even in polymers, when pertinent graphical representations give straight lines over a wide range of current. But, then, arises the problem of expressing the preexponential factor in PF laws. Usually this factor is considered as independent of $F$ in cases, irrelevant to Schottky mechanism, where extended linear behaviour obtains in a $\left(\lg I, F^{1 / 2}\right)$ plot. However, a wealth of other possibilities avails in literature. For example, Jonscher [6] evokes two possible assumptions as to the fate of electrons out of centres. First, electrons can be re-trapped by nearest downward 
centres. The author obtains so a pre-exponential factor proportional to $F^{-1 / 2}$, with three-dimensional wells. Secondly, Jonscher assumes that the free carriers may drift during a constant time before retrapping. A proportionality to $F^{1 / 2}$ is then found. Besides following Hill [5], the drift velocity of electrons, in completely amorphous materials, should be proportional to $F^{1 / 2}$ as a result of a random diffusion process. Likewise, Hall [7] proposes for amorphous materials a pre-exponential factor proportional to $F^{-3 / 2}$ or to $F^{-3 / 4}$ corresponding respectively to hopping-type transitions and to diffusion out of centres. Moreover the two current components, simultaneously present, exhibit dependences of respective forms $\exp \beta \sqrt{F} / 2 k T$ and $\exp \beta \sqrt{F} / k T$ (see (vi) below). But the problem of knowing in what $F$ and $T$ combined ranges each one is dominant, is not straightforward. It is to be stressed here that, as a consequence, a fair choice of a graphical representation is not free from ambiguity. This could explain why many experimentalists do not sharply discriminate $\left(\lg I, F^{1 / 2}\right)$ and $\left(\lg I / F, F^{1 / 2}\right)$ as $\mathrm{PF}$ plots.

(v) Coulombic centres are mostly considered (donors). Despite the fact that they can hardly be accepted when deep levels are concerned, the range of application of PF theories is usually extended up to activation energies in excess of $0.5-0.6 \mathrm{eV}$. These values are that given by Chang et al. [8]. But many experimentalists use to consider activation energies, deduced experimentally from PF analysis, ranging up to $1.5 \mathrm{eV}$ (Lovell [9]), $1.6 \mathrm{eV}$ (Hancomb and Kaahwa [10]), or even $2 \mathrm{eV}$ (Vollmann [11]), this author taking, however, such a high value in a simulation process only. If this range had to be limited to much lesser values, we shall show in the following that PF saturation, to be defined hereafter, would reduce significantly the uppermost field beyond which PF effect ceases to apply. However, potentials steeper than the hydrogenic potential are sometimes introduced in PF theories. They belong to two main kinds : potentials in $r^{-n}(n>1)$ originating in multipolar defects (Arnett and Klein [12]), or screened Coulomb potentials (Fritzsch and Bobe [13]). But then, the classical PF law is modified. For example, with centres of the first kind, I becomes proportional to $\exp \left(K F^{\mathrm{n}}\right)$ with $\frac{1}{2}<\mathrm{n}<1$ and $\mathrm{n} \rightarrow 1$ as $n \rightarrow \infty$. The impurity potential is then less perturbed by the applied field, and saturation is delayed to higher fields.

(vi) Donors are considered either alone (Frenkel [1], O'Dwyer [14]), or more or less compensated by acceptors. In addition traps, neutral when empty, are sometimes introduced, independently of acceptor sites. When acceptors are supposed to exist, they are always conjectured as being entirely filled with electrons from donors, whatever $F$ and temperature
$T$. Authors adopt usually various compensation rates and distributions of neutral traps, seemingly as a mean of providing, in a $\left(\lg \sigma, F^{1 / 2}\right)$ plot, slope adjustment to any values between $\beta / 2 k T$ (donors alone) and $\beta / k T$, with $\beta=\left(e^{3} / \varepsilon \varepsilon_{0}\right)^{1 / 2}$ (Simmons [15] ; Mark and Hartman [16] ; Yeargan and Taylor [17]). This can afford an opportunity of application of PF theories, to cases less simpler than that treated originally by Frenkel, in contradistinction to Schottky effect. It is to be emphasized at this point, that a large amount of data results in slopes smaller than expected, leading thus to rather large values of the involved dielectric constant $\varepsilon$. Alternatively, miscellaneous trapping can also be considered as a way likely to succeed in an appropriate location of the Fermi energy. Besides following Jonscher and Ansari [18], neutral traps in amorphous semiconductors can modify the transport processes of electrons, excited from donors by the field, in such a way that an effective temperature introduces in the PF equation.

(vii) One donor level alone is generally stated. Additional levels are sometimes introduced (Mark and Hartman [16], but they are then supposed to lie far below the Fermi energy, so that they remain completely filled over the entire ranges of $F$ and $T$, and does not supply electrons to conduction band. This hypothesis seems to be supported by the fact that complementary experiments such as thermostimulated currents are in favour of a one-level donor distribution (Servini and Jonscher [19]). But we shall show in a following paper that these are not unquestionable proofs.

(viii) Moreover, the various PF models can be roughly subdivided into one-dimensional (PF-1D) and three-dimensional (PF-3D) models, the latter taking account of the direction of electron emission. The three-dimensional models differ from one another by the way in which the probability of electron ionization in the reverse direction is considered. For Jonscher [20], this is a negligible quantity; for Hartke [21], it does not depend on field. Hill [5], as well as Connell et al. [22], determine this probability as if the barrier enhancement in the reverse direction were equal to the barrier lowering in the forward direction. Ieda et al. [23], in addition, truncate the potential well at a level $\varphi \sim k T$ below the conduction band. They obtain then two different PF laws, appropriate for low and high fields respectively. In addition, it can be noted that authors do not agree on whether the two current components, in the forward and reverse directions, have to be summed or subtracted. This seems in relation with a too much imprecise statement of the fate of excited electrons. Such difficulty is apparently dropped in a treatment by Pai [24]. This author uses Onsager theory of Brownian motion, under the influence of a field, of particules out of coulombic potentials (Onsager [25, 26]). 
The above review shows that, concerning PF effect, every ambiguity is far from being removed, both from a theoretical point of view and from an examination of the conditions in which experimentalists use to implement PF theories. An extensive study of PF effect can be found in [27]. For the sake of simplicity, this paper is dealt with PF-1D case only.

\section{PF saturation requirement.}

A number of the above models lead to relations tending asymptotically towards Ohm's law at low fields. But the essential feature, in view of the following developments, is that they are all indefinitely increasing functions, eventually expressible as :

$$
\sigma_{\text {H.Field }} \propto \exp \left(\frac{\beta \sqrt{F}}{k T}\right)=\exp (\alpha)
$$

However, PF effect proceeding from a donor emptying process would inevitably result in a situation where centres become completely ionized. Thus the notion of PF saturation follows directly as a consequence of the model. But despite its obviousness, this notion is completely missing in literature. As we shall show below, this comes from the simplification, adopted steadily by authors, following which Boltzmann function can be taken as the always convenient approximation of Fermi-Dirac function. Moreover, as shown in figure 1 , the coulombic well model loses any physical meaning for sufficiently high field strenghts. The potential barrier of such a well, of depth $\Phi$, is reduced in the field direction to the magnitude $\Phi-\beta \sqrt{F}$ (curve a). The barrier vanishes (curve b) when $F$ amounts to :

$$
F=F_{\mathrm{s}}=(\Phi / \beta)^{2}
$$

$F_{\mathrm{s}}$ is called field of saturation.

Sites are then necessarily fully ionized, and the density of electrons in conduction band is a maximum. For $F>F_{\mathrm{s}}$ the potential turning-point is located below the ground state of centres. No physical meaning can then be affected to centres so soundly perturbed by the field.

But the question arises then to know the reasons why PF saturation has apparently never been put forward experimentally. It can be thought that, in effective experiments, $F$ remains always smaller than $F_{\mathrm{s}}$. But determination of some orders of magnitude shows that this is not necessarily the case. For example, with $\varepsilon=2.2$ and for a site of depth $\Phi=1 \mathrm{eV}, F_{\mathrm{s}}=3.8 \times 10^{8} \mathrm{~V} \cdot \mathrm{m}^{-1}$, a value lesser or comparable to the breakdown field $F_{\mathrm{d}}$ of some insulators. Thus, for relatively shallow donor sites, the condition $F_{\mathrm{s}}<F_{\mathrm{d}}$ may be fulfilled. In such a case, saturation is likely to occur ; and particularly when a distribution of donors in energy is considered. Why then no experimental proof of PF saturation exists in literature. We believe that, in absence of the theoretical concept of saturation, experimentalists liable to have observed such an effect, would have preferentially chosen other mechanisms than PF effect to interpret their data.

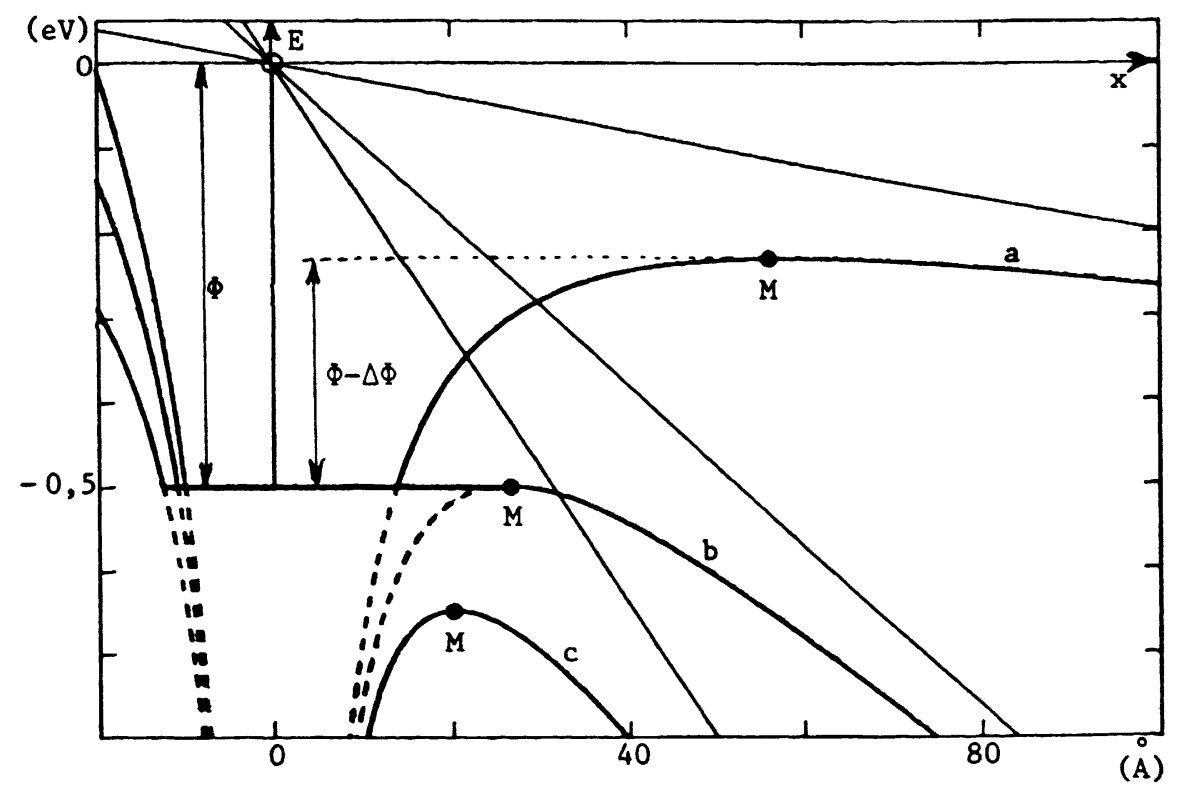

Fig. 1. Evolutions of the field-induced coulombic well profiles (a) $F=2 \times 10^{7} \mathrm{~V} \cdot \mathrm{m}^{-1} ;(\mathrm{b}) F=F_{\mathrm{s}}=9.52 \times$ $10^{7} \mathrm{~V} \cdot \mathrm{m}^{-1} ;$ (c) $F=1.6 \times 10^{8} \mathrm{~V} \cdot \mathrm{m}^{-1} ; \varepsilon=2.2 ; \Phi=0.5 \mathrm{eV}$. 
3. Effective determination of saturation, when compensation is present.

3.1 DESCRIPTION OF THE MODEL, AND CALCULATION. - The concept of saturation being now well stated, a complete calculation is needed. In order to give a little more general approach, we develop the case where compensation by acceptor levels is present in any proportion. No other kind of traps are supposed to exist. This latter hypothesis is made to simplify calculations at the best, and as we do not think it to be an essential one. Anyway it can be re-introduced easily to give a little more sophisticated model.

Five hypotheses are then made (Blakemore [28]), and lead to figure 2 .

(i) Acceptor levels are situated well below the Fermi level, so that they remain completely filled, whatever $F$ and $T$.

(ii) A single donor level exists, at a depth $\Phi$ below the conduction band $(\Phi \gg k T)$.

(iii) The donor density $N_{\mathrm{d}}$ is greater than the density $N_{\mathrm{a}}$ of acceptors. We define then a compensation rate as the ratio $q=N_{\mathrm{d}} / N_{\mathrm{a}}(q>1)$. Ionized donors and acceptors are supposed to lie far apart, so that the two species do not perturb significantly one each other. This hypothesis could be difficult to maintain when $q \rightarrow 1$ and $N_{\mathrm{d}}$ is very large. However, it is to be noticed that, when large values are given to $N_{\mathrm{d}}$ and $N_{\mathrm{a}}$ this aims mainly in describing the limiting behaviour of the studied functions, sometimes at the risk of blurring somewhat the pertinence of the model.

(iv) Boltzmann function can be used only to describe the free electron density. Hence

$$
n=N_{\mathrm{c}} \exp \left(-\Phi_{\mathrm{F}_{\mathrm{n}}} / k T\right)
$$

(v) Fermi-Dirac statistics is used to define the population of filled donors in the gap. Besides being necessary to express saturation, this allows us to avoid, contrary to previous treatments, any at hand hypothesis about the respective positionings of donor level and of Fermi level.

So, when an external field is applied, we can write :

$$
n+N_{\mathrm{a}}=\frac{N_{\mathrm{d}}}{1+\exp \left(\frac{\Phi-\beta \sqrt{F}-\Phi_{\mathrm{F}_{\mathrm{n}}}}{k T}\right)}
$$

In this equation, it is supposed that the degeneracy factor $g$ for electrons in donor sites equals 1 . This is only a simplifying assumption, introduced in order that asymptotic expressions of (4a) could be quantitatively in accordance with the Boltzmann functions found in literature. Now, a spin degeneracy factor $g=1 / 2$ should avail as pre-exponential factor in (4a), for a monovalent impurity. But, taking $g=1$ does not affect significantly the analysis below. Then, substituting in this equation $n / N_{\mathrm{c}}$ for $\exp \left(-\Phi_{\mathrm{F}_{\mathrm{n}}} / k T\right)$, leads readily to :

$$
\begin{aligned}
\left(\frac{n}{N_{\mathrm{c}}}\right)^{2}+\frac{n}{N_{\mathrm{c}}}( & \left.\exp (-\eta+\alpha)+\frac{s}{q}\right)- \\
& -s \frac{q-1}{q} \exp (-\eta+\alpha)=0
\end{aligned}
$$

with $\eta=\Phi / k T ; \alpha=\beta \sqrt{F} / k T ; s=N_{\mathrm{d}} / N_{\mathrm{c}}$ and $N_{\mathrm{c}}=2\left(2 \pi m^{*} k T / h^{2}\right)^{3 / 2}=K_{\mathrm{c}} T^{3 / 2}$. The relative density $n_{\mathrm{r}}=n / N_{\mathrm{c}}$, of electrons in conduction band, can then be expressed by :

$$
\begin{aligned}
n_{\mathrm{r}} & =\frac{1}{2} \mathrm{e}^{-\eta+\alpha}\left(1+\frac{s}{q} \mathrm{e}^{\eta-\alpha}\right) \times \\
& \times\left(\sqrt{1+4 s \frac{q-1}{q} \frac{\mathrm{e}^{\eta-\alpha}}{\left(1+\frac{s}{q} \mathrm{e}^{\eta-\alpha}\right)^{2}}}-1\right)
\end{aligned}
$$

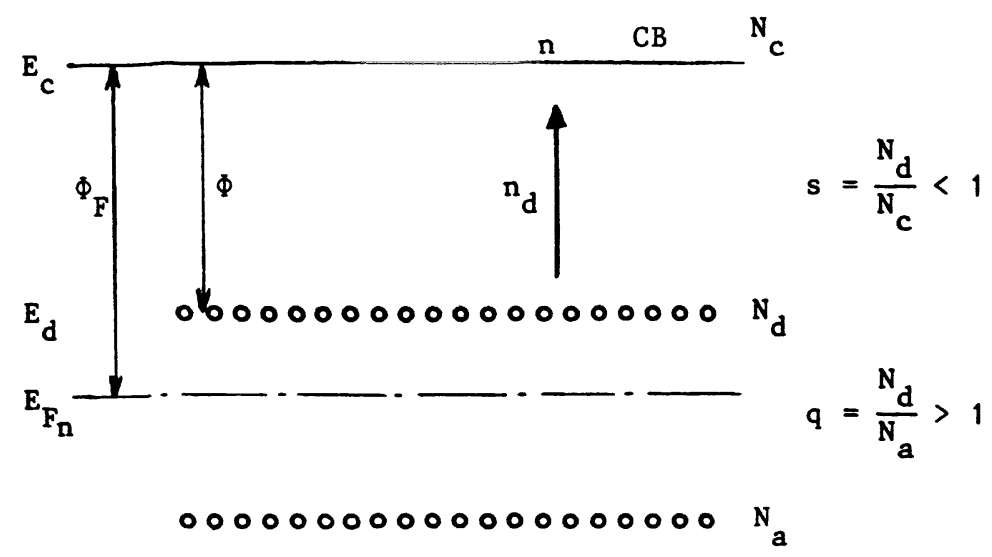

Fig. 2. - Representation of energy levels in the gap. Relative positions of $E_{\mathrm{d}}$ and $E_{\mathrm{F}}$ are arbitrary. 
or

$$
\begin{aligned}
n_{\mathrm{r}}= & \frac{2 s \frac{q-1}{q}}{\left(1+\frac{s}{q} \mathrm{e}^{\eta-\alpha}\right) \times} \\
& \times\left(\sqrt{1+4 s \frac{q-1}{q} \frac{\mathrm{e}^{\eta-\alpha}}{\left(1+\frac{s}{q} \mathrm{e}^{\eta-\alpha}\right)^{2}}}+1\right)
\end{aligned}
$$

The quasi-Fermi level position, under an applied field, follows immediately :

$$
-\Phi_{\mathrm{F}_{\mathrm{n}}}=k T \operatorname{Ln}\left(n_{\mathrm{r}}\right) \text {. }
$$

\subsection{Simulated BEHAVIOUR IN TERMS OF FIELD $F$.}

- In this sub-section we analyse the response to an electric field, of a dielectric representable by the above model. We chose for that a large set of plausible values for parameters $s, q$, and $\eta$, and for the variable $\alpha$. We determined first variations with field of $n_{\mathrm{r}}(\alpha)$, and then that of the quasi-Fermi level $\Phi_{\mathrm{F}_{\mathrm{n}}}(\alpha)$.

3.2.1. Variations of $n_{\mathrm{r}}$ versus field. - Relative variations of $\lg n_{\mathrm{r}}$ in terms of $\alpha$, are given in figure 3 , with $\eta=40$, and for $s=10^{-1}$ and $s=10^{-6}$. $\alpha$ ranges from zero to $\alpha_{\mathrm{s}}=\eta$ (saturation). $q$ is the leading parameter ; it is varied by series of ten from 1.04 to $10^{7}$. This allows simulation of cases passing progressively from nearly fully compensated materials, to non-compensated materials. Though calculations are made in $\alpha$, figure 3 is also scaled in $\alpha_{\mathrm{p}}, \alpha_{\mathrm{p}}$ being the relative field-induced potential lowering when non-coulombic centres are involved. Thus, plotting becomes independent of the particular form of the effective relationship relating $\alpha_{\mathrm{p}}$ to $F$.
For example, when a dipolar centre is concerned a $F^{2 / 3}$ law is substituted for the classical $F^{1 / 2}$ law.

Saturation is very apparent in the figure. It begins at fields the weaker the weaker $s$. Actually, PF saturation, as defined above, sets in only for $\alpha_{\mathrm{s}}=40$. The observed precocious curve levelling is apparently associated with the relative depletion of donor densities, the maximum of $n_{\mathrm{r}}$ amounting necessarily to $\left(N_{\mathrm{d}}-N_{\mathrm{a}}\right) / N_{\mathrm{c}}=s(q-1) / q$. This levelling off is directly dependent on Fermi-Dirac function ; it disappears when Boltzmann's function is used (dashed higher parts of curves), for it leads to an equation like (8). However, in some cases like that represented by curve $h$, function (5) is still growing for $\alpha_{\mathrm{p}}=\alpha_{\mathrm{ps}}$. This should imply that centres are not yet fully empty, at the time where they are losing physical meaning. But one cannot agree with such an idea, as it is irrelevant with Fermi-Dirac statistics. Now, it will be seen below (see Fig. 4) that a certain degree of degeneracy takes place in such cases. So that hypothesis (iv), and consequently equation (5a), becomes invalid in the vicinity of $\alpha_{\text {ps }}$. Then the only right procedure would consist in substituting the true analytical expression of $n_{\mathrm{r}}$ for $\exp \left(-\Phi_{\mathrm{F}_{\mathrm{n}}} / k T\right)$. But, strictly speaking, this is impossible as no analytical expression exists for the relevant Fermi-Dirac integral. Nevertheless, some approximate forms have been derived, available in ranges of energy more or less restricted upwards. In the present case, and for the sake of simplicity, it is permissible to adopt the approximate form given by Ehrenberg [29] :

$$
n_{\mathrm{r}}=\left(C+\exp \left(\frac{\Phi_{\mathrm{F}}}{k T}\right)\right)^{-1}
$$

Following Blakemore [28], the most appropriate value for constant $C$ is $C=0.27$ for parabolic bands.

Then $n_{\mathrm{r}}$ takes the form :

$$
n_{\mathrm{r}}=\frac{1}{2} \frac{1+\frac{s}{q} \mathrm{e}^{\eta-\alpha}+s C \frac{q-1}{q}}{\mathrm{e}^{\eta-\alpha}-C}\left(\sqrt{1+4 s \frac{q-1}{q} \frac{\mathrm{e}^{\eta-\alpha}-C}{\left(1+\frac{s}{q} \mathrm{e}^{\eta-\alpha}+s C \frac{q-1}{q}\right)^{2}}}-1\right) .
$$

It is evident that (7) transforms into (5a) if $C$ vanishes. We did not attempt a comparative simulation of this equation.

Figure 3 shows also that for identical $q$, and for sufficiently low $\alpha_{\mathrm{p}}$ values, the obtained curves murge in, and become independent of $s$.

In order to compare the present curve shapes to that given in literature, we recall that, following Yeargan and Taylor [17], the various PF-1D pub- lished laws can take the general form :

$$
n=K \exp \left(\frac{\beta \sqrt{F}}{m k T}\right)=K \exp \left(\frac{\alpha}{m}\right)
$$

$m$ being a «slope parameter » ranging from 1 to 2 . The case $m=1$ is labelled hereafter as « usual »PF effect ; while the case $m=2$ is called « original »PF effect, as it is dealing readily with Frenkel formulation. 


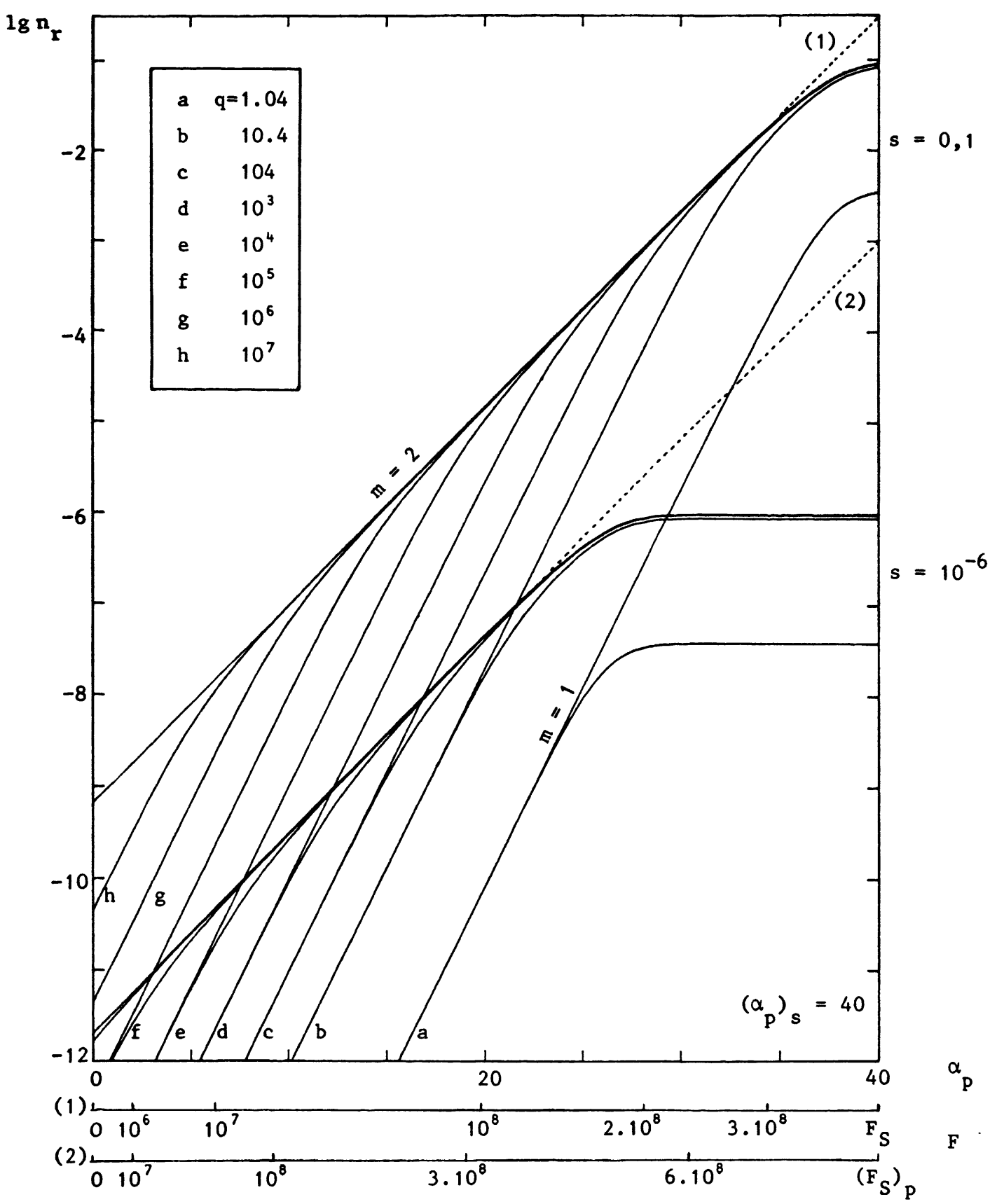

Fig. 3. - Variations of $\lg n_{\mathrm{r}}$ versus $\alpha_{\mathrm{p}}$, with $\eta=40$. The two field scalings (in $\mathrm{V} . \mathrm{m}^{-1}$ ) correspond respectively to : (1) $\alpha_{\mathrm{p}}=\alpha=\frac{\beta \sqrt{F}}{k T}$ (usual PF effect with $\varepsilon=2.2, T=300 \mathrm{~K}, \Phi=1.04 \mathrm{eV}$ ); (2) $\alpha_{\mathrm{p}}=\frac{\left(\beta^{2} e \ell\right)^{1 / 3}}{k T} F^{2 / 3}$ (PF effect for dipolar wells, with wells : $\varepsilon=2.2, \ell=1 \AA, T \cong 173 \mathrm{~K}, \Phi=0.6 \mathrm{eV}$ ). Dotted lines represent the Boltzmann function, for large $q$ values only.

Returning to figure 3 allows then to make the following remarks. When a practically full compensation is achieved ( $q \sim 1$, curve a), $n$ follows out a « usual »PF law, until saturation begins to set in. When compensation is negligibly small $\left(q=10^{7}\right.$, curve $h$ ), the initial «usual » PF regime gives up rapidly, followed by an « original » $\mathrm{PF}$ regime. For intermediate values of $q$, the transition field from the first regime to the second one, is a decreasing function of $q$.
We conclude from this that whenever donors and acceptors only are supposed to exist, a pure « usual » $\mathrm{PF}$ effect is found for full compensation, whilst a pure « original » PF effect appears in the case of no compensation at all. Thus, the pioneering work of Frenkel is well corroborated apart from occurrence of saturation. But the analysis shows inversely that authors like O'Dwyer [14] were not founded to introduce the « usual » PF effect, when donors only are present. 


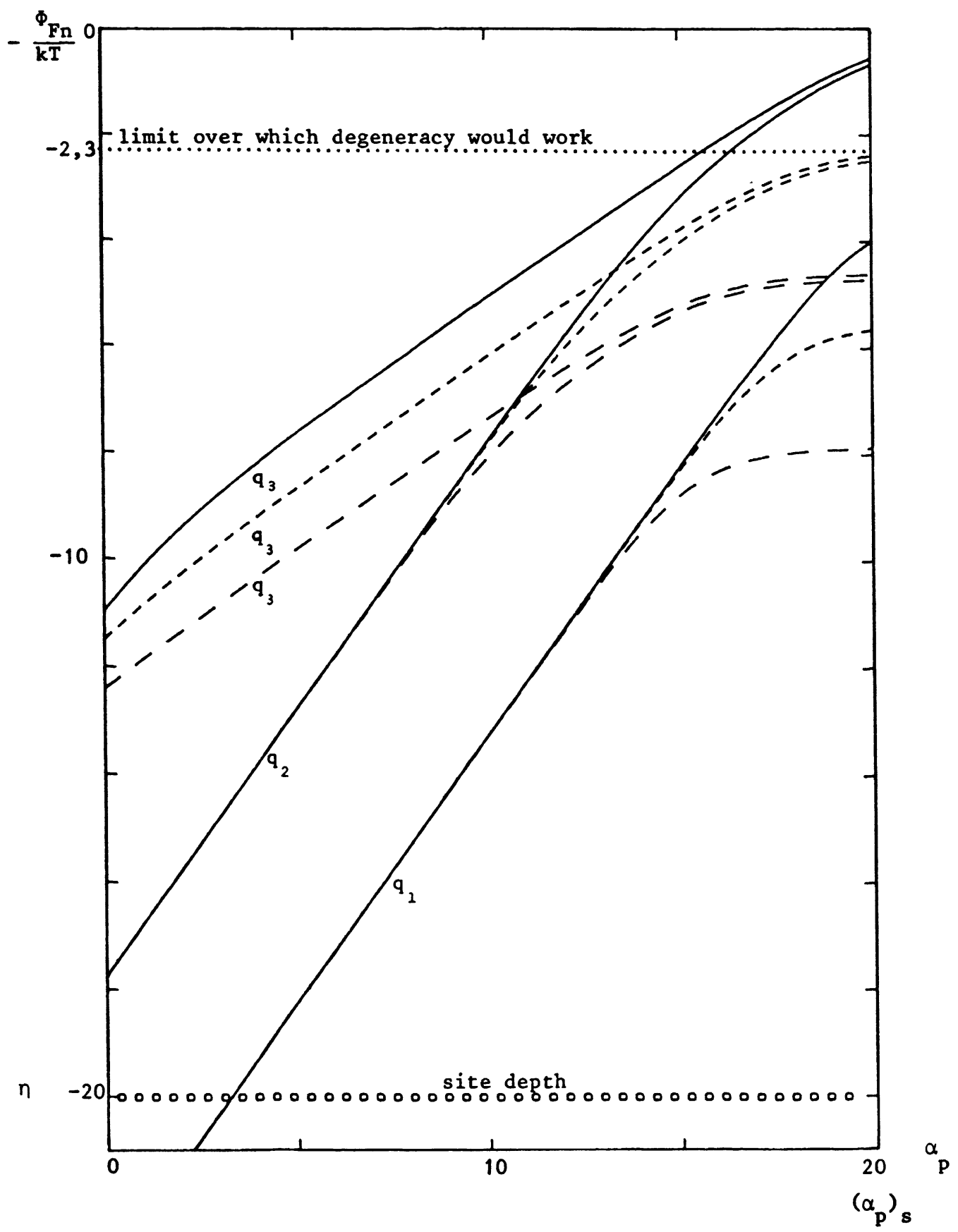

Fig. 4. - Relative variations of $\Phi_{\mathrm{F}_{\mathrm{n}}} / k T$ versus $\alpha_{\mathrm{p}}$, for various values of $s$ and $q$, and with $\eta=20$. (- - - - ) $s=0.01 ;(-\cdots-) s=0.1 ;(\longrightarrow) s=0.9 ; q_{1}=1.04 ; q_{2}=10.4 ; q_{3}=10^{4}$.

Finally our approach, based upon Fermi-Dirac statistics, leads to two important results. (i) in general conditions, the « usual »PF effect is present together with the « original » PF effect; each can appear alone, only in limiting cases. (ii) PF saturation is a fundamental notion, « consubstantial » to barrier-field-lowering.

Our model could be completed by introducing neutral traps, insensitive to the field, as did Mark and Hartmann. This would give two more parameters, which undoubtedly, would bring opportun- ities to shift as desired the saturation threshold towards high field strengths.

3.2.2 Quasi-Fermi level evolutions with field. Curves of figure 4 give the relative variations of the quasi-Fermi level $\Phi_{\mathrm{F}_{\mathrm{n}}} / k T$, in terms of $\alpha_{\mathrm{p}}$, that is for the present purpose, in $\alpha$. Three sets of curves are drawn, corresponding to the three values of $s: 0.01$, $0.1,0.9$. Each set involves three curves obtained respectively for $q=1.04, q=104$ and $q=10^{4}$. The bottom of conduction band is taken as zero-poten- 
tial. Depth of centres is represented by the opencircle horizontal line $(\eta=20)$, which, for roomtemperature, corresponds to $\Phi \cong 0.5 \mathrm{eV}$.

It is apparent in the figure that, when compensation is nearly complete $(q \sim 1)$ the quasi-Fermi level lies below the ground-state of coulombic centres, for the lowest field strengths. Hence, FermiDirac statistics are needed to describe the fieldinduced ionization process. But for weak compensation $(q \gg 1)$, the reverse is true. Then Boltzmann function can be used as a valid approximation, for every magnitude of $\alpha$, except in the vicinity of saturation. And $n_{\mathrm{r}}$ takes the form :

$$
n_{\mathrm{r}}=\frac{s}{2 q}\left(\sqrt{1+4 \frac{q^{2}}{s} \exp (-\eta+\alpha)}-1\right)
$$

Simulation shows that this equation gives precisely the whole straight lines (1) and (2) of figure 3.

An interesting additional remark can be made, from a methodological view-point. If equation (5a) is given in its approximate form, when $\frac{s}{q} \mathrm{e}^{\eta-\alpha} \gg 1$, one obtains :

$$
n_{\mathrm{r}}=\frac{s}{2 q}\left(\sqrt{1+\frac{4 q(q-1)}{s} \mathrm{e}^{-\eta+\alpha}}-1\right)
$$

instead of equation (8a). Significant departures between these equations can then be expected, when a nearly full compensation is achieved $(q \rightarrow 1)$. This example shows how misleading formulations can, sometimes, result from untimely simplifications of a model.

Moreover, figure 4 shows that the quasi-Fermi energy $E_{\mathrm{F}_{\mathrm{n}}}$ is an increasing function of $F$. As $F$ is increased, $E_{\mathrm{F}_{\mathrm{n}}}$ tends toward a limit, reached the more rapidly the weaker s. For a very high density of donors $(s=0.9)$, and with very poor compensation $\left(q=104, q=10^{4}\right)$, a certain degree of Fermi-level degeneracy begins to set in at higher fields. From the above considerations about Fermi-Dirac integrals, the quasi-Fermi level positions, given by figure 4 , are not very accurate, in the range of saturation.

\section{Study using a « sliding » variable, $1-\alpha / \eta$.}

In the preceding sub-sections, we considered $\alpha$ as the direct (reduced) variable, and $\eta$ as a parameter, characteristic of the material under investigation. But if we look at equations (5), (7) and (8), we observe they depend actually on the function $\exp (\eta-\alpha)$. Thus more generality can be achieved, if we consider, instead of $\alpha, \eta-\alpha$ or rather

$$
X=1-\frac{\alpha}{\eta}=1-\frac{\alpha}{\alpha_{\mathrm{s}}}=1-\left(\frac{F}{F_{\mathrm{s}}}\right)^{1 / 2}
$$

Or quite generally

$$
X=1-\frac{\alpha_{\mathrm{p}}}{\eta}=1-\frac{\Delta \Phi_{\mathrm{p}}}{\Phi}
$$

$X$ is then a relative "sliding " variable, $\Delta \Phi_{\mathrm{p}}$ being the barrier lowering for a non-coulombic PF effect.

4.1 VARIATIONS OF CONDUCTIVITY. - Figure 5 is a plot of $\lg \sigma$ against $X$, for a set of $q$ values varying by series of ten from 1.04 to $10^{6}$. Three families of curves are defined, by choosing three values of the leading parameter $s\left(10^{-1}, 10^{-4}, 10^{-6}\right)$. A doubly scaled abscissa is drawn, to give simultaneously $X$ and $\eta X$.

Curves of figure 5 are symmetrical, with regard to a vertical axis, to the corresponding ones of figure 3 . But figure 5 allows a general discussion more easily. In this figure, it is clear that an essential feature is the choice of an « observation window ». From that, it appears that various conclusions are possible, in the framework of the aforementioned PF theories, depending on the behaviour of a given set of experimental data. Consider for instance an ensemble of coulombic wells, of depth $\Phi \cong 1 \mathrm{eV}$. Suppose moreover a field strength variation from $2 \times 10^{6} \mathrm{~V} . \mathrm{m}^{-1}$ to $2 \times 10^{8} \mathrm{~V} \cdot \mathrm{m}^{-1}$. Then, if $\varepsilon=2.2$ and $T=300 \mathrm{~K}$, the "observation window » is limited by $\eta X=12$ (vertical dotted-line $\mathrm{A}$ ), and $\eta X=37.2$ (dotted-line B), or by $X=0.30$ and $X=0.93$. Then our model shows that, depending on magnitudes of $s$ and $q$, an experimentalist can find mainly three kinds of curves. If a curve similar to (1) is obtained, he should infer that his data are relevant to an « original» PF effect. Likewise, curve (2) would give a « usual » PF effect. If a curve similar to (3) is found, he would incline towards a less simple explanation, where for example, space charge effects are taken into consideration (Frank and Simmons [2] ; Murgatroyd [4]).

Finally, as a great deal of crossed choices can be made among the various parameters, it becomes clear that our theory succeeds in reducing to a unified approach many of the existing PF theories.

4.2 EVOLUTION OF THE «SLOPE PARAMETER» $m$. - In the general case of equations (5), $m$ is defined as the inverse of the local slope of curves of figure 3 .

That is :

$$
m=-\left(\frac{\mathrm{d}(\operatorname{Ln}(n))}{\mathrm{d} \alpha}\right)^{-1}
$$

Differentiating equations (5a) or (5b), leads then to :

$$
m=-\frac{n_{\mathrm{r}}-n_{1}}{n_{1}+\mathrm{e}^{-\eta X}}
$$

In this equation, $n_{\mathrm{r}}$ and $n_{1}$ are the relative solutions, positive and negative respectively, of equation (4b). 


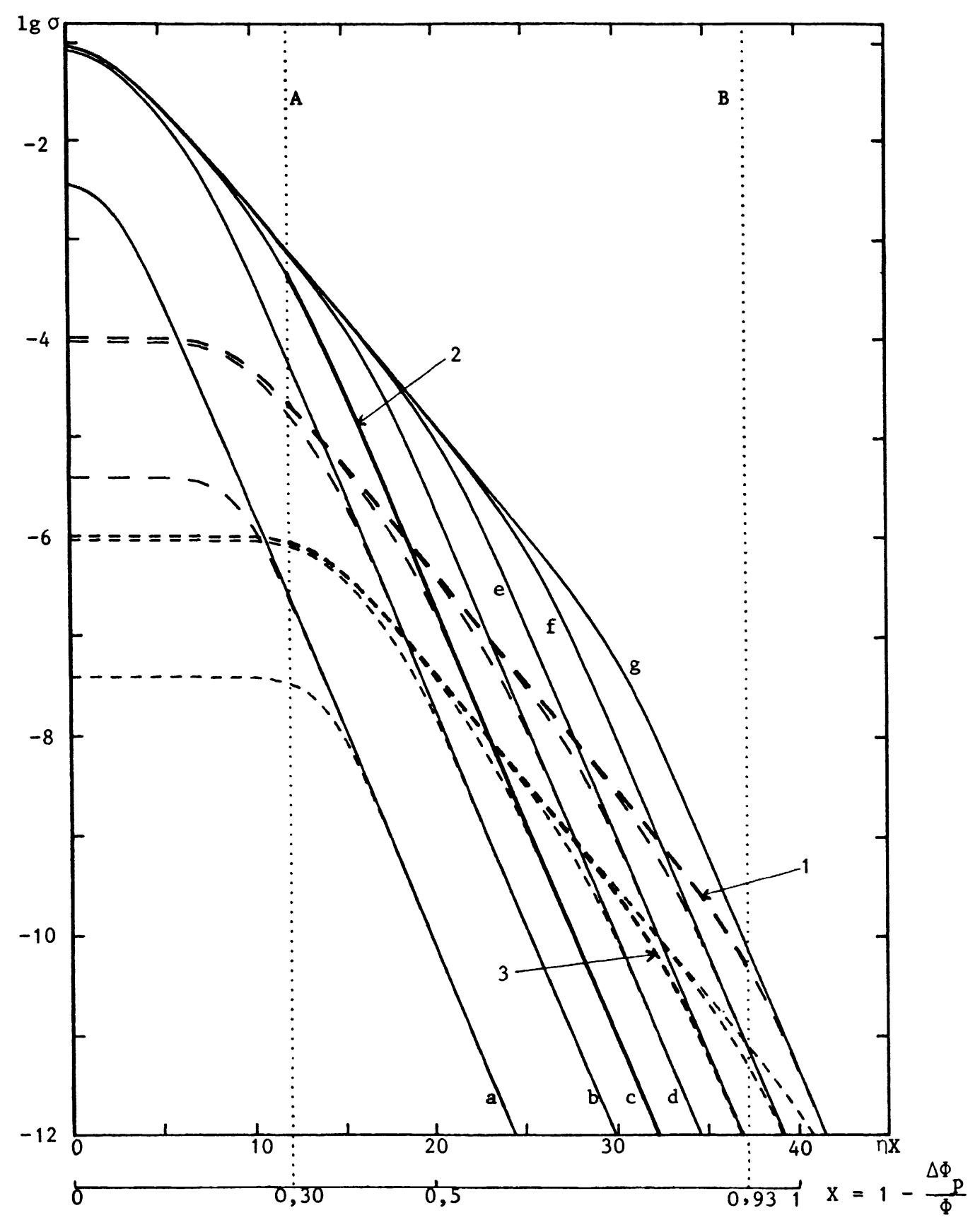

Fig. 5. - Variations in arbitrary units, of $\lg \sigma$ against $\eta X=\eta\left(1-\frac{\alpha_{\mathrm{p}}}{\eta}\right)(\longrightarrow)$ $s=10^{-4}$; (- - - - $s=10^{-6}$. Labelling of curves is that of figure 3. Vertical dotted lines A and B delimit an « observation window » as quoted in the text.

Given equation (10), we are allowed to delimit possible intervals of variation for $m$ in terms of $\eta X$. In figure 6 , curves are shared out amoung three families associated with three values of $s: 10^{-1}$, $10^{-2}$ and $10^{-6}$.

It appears that, when compensation is very weak, $\boldsymbol{m}$ passes through the stationary values 1 and 2 , which gives the straight portions of curves in figure 3 . While, for heavy compensation, $m$ goes rapidly to 1 , so that the «usual »PF effect is obtained over the most part of the $X$ interval. Horizontal dotted lines $(m=1$ and $m=2$ ) are drawn to emphasize the behaviour of $m$ resulting from Boltzmann function. An additional horizontal line, placed between 1 and 2 , would correspond to cases evoked by Yeargan and Taylor [17], and Pai [24]. Figure 6 shows in addition, that values of $m>2$ can be obtained, over a more or less extended interval. But then, $m$ does not remain constant. Hence, a lot of experimental data, interpreted in terms of classical PF effects, 


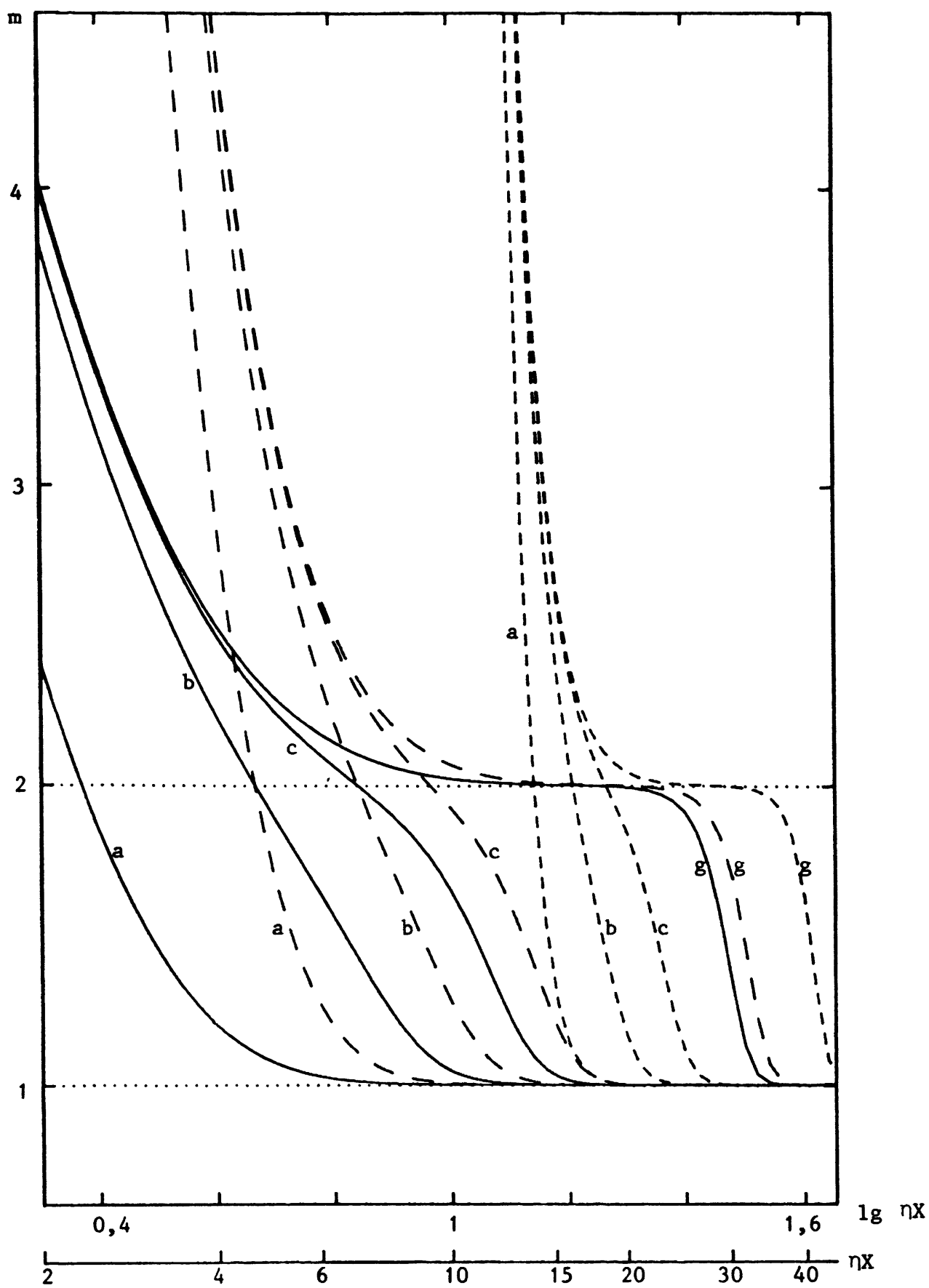

Fig. 6. - Variations of $m$ as a function of $\eta X$. $10^{-6}$. Same labelling of curves as in figure 3 .

with $m>2$, do not seem to be relevant to our theory. A better relevance will be achieved when distributions of donors in energy will be accounted for.

\section{Conclusion.}

Our approach allowed us to show, in the domain of theoretical studies of PF effect where everything seemed to have been said for a long time, fundamental questions needed to be specified. As an extension of PF theories our development refers only to strictly isolated coulombic centres, thermally connected to the host lattice through Fermi-Dirac statistics. In the present paper, we went thouroughly into the essential, but still cast aside or misunderstood, notion of PF saturation. Doing this led us to put forward the misleading character of some over-simplifications, even when apparently justified by ad hoc hypotheses. This is, particularly, the case for the description of trap populations by Boltzmann function, substituted 
rather universally for Fermi-Dirac function. Using Fermi-Dirac statistics have resulted in a more general approach of PF effect, following which the so-called « original » and « usual » PF effects appear only as, limiting, peculiar aspects. Moreover, introducing a "sliding " variable allowed us to show, through the choice of an «observation window», that a large diversity of curves were interpretable, from an experimental point of view, by our present PF theory.

\section{Acknowledgements.}

We are very indebted to Professor R. Coelho, from the Ecole Supérieure d'Electricité, Gif sur Yvette, for many helpful discussions and suggestions about the present work.

\section{References}

[1] Frenkel J., Phys. Rev. 54 (1938) 647-8; Sov. Phys. Techn. Phys. 5 (1938) 685.

[2] Franck R. I., Simmons J. G., J. Appl. Phys. 38 (1967) 832-40.

[3] Simmons J. G., Phys. Rev. 166 (1968) 912-20.

[4] Murgatroyd P. N., J. Phys. D 3 (1970) 151-6.

[5] Hill R. M., Philos. Mag. 23 (1971) 59-86.

[6] JonsCher A. K., Thin Solid Films 1 (1967) 213-34.

[7] Hall R. B., Thin Solid Films 8 (1971) 263-71.

[8] Chang C. Y., Hsu W. C., Wang S. J., Hau S. S., J. Appl. Phys. 60 (1986) 1042-5.

[9] Lovell R., J. Phys. D 7 (1974) 1518-30.

[10] Hanscomb J. R., KaAhwa Y., J. Phys. D 12 (1979) 587-99.

[11] VollmanN W., Phys. Status Solidi a 195 (1974) 195203.

[12] Arnett P. C., Klein N., J. Appl. Phys. 46 (1975) 1399-1400.

[13] Fritzsch L., Bobe W., Phys. Status Solidi b 58 (1973) K49-K52.

[14] O'DWYER J. J., The theory of electrical conduction and breakdown in solid dielectrics (Clarendon Press, Oxford) 1973.

[15] Simmons J. G., Phys. Rev. 155 (1967) 657-60.
[16] Mark P., Hartmann T. E., J. Appl. Phys. 39 (1968) 2163-4.

[17] Yeargan J. R., Taylor H. L., J. Appl. Phys. 39 (1968) 5600-4.

[18] Jonscher A. K., Ansari A. A., Philos. Mag. 23 (1971) 205-23.

[19] Servini A., Jonscher A. K., Thin Solid Films 3 (1969) 341-65.

[20] Jonscher A. K., J. Phys. C 3 (1970) L159-62.

[21] Hartke J. L., J. Appl. Phys. 39 (1968) 4871-3.

[22] Connell G. A. N., Camphausen D. L., Paul W., Philos. Mag. 26 (1972) 541-51.

[23] Ieda M., Sawa G., Kato S., J. Appl. Phys. 42 (1971) 3737-40.

[24] Pai D. M., J. Appl. Phys. 46 (1975) 5122-6.

[25] Onsager L., J. Chem. Phys. 2 (1934) 599-615.

[26] Onsager L., Phys. Rev. 54 (1938) 554-7.

[27] Pillonnet A., Thesis, Université Lyon I (1988).

[28] BLAKEMORE J. S., International series of monographs on semi-conductors, Vol. 3 (Pergamon Press) 1962.

[29] Ehrenberg, Proc. Phys. Soc. London A 63 (1950) 75. 